\title{
Challenges of Glycemic Control in COVID-19 Patients with Diabetes Mellitus in Resource-poor Settings
}

\author{
Esan, A., Azeez, T. A.*, \\ Endocrinology Unit, Department of Medicine, University College Hospital, Ibadan, Nigeria \\ Corresponding author: Dr. Azeez, T. A.; adegokegalaxy@yahoo.com
}

Received 16 July 2020;

Accepted 02 August 2020;

Published 14 August 2020

\begin{abstract}
Background and objectives: Coronavirus disease 2019 is now a global pandemic affecting most countries in the world. Some conditions, such as diabetes mellitus, are associated with increased morbidity and mortality among COVID-19 patients. Poor glycemic control has been associated with the risk of contracting COVID-19 and it is also poor prognostic factors among patients infected. Therefore, all efforts should be focused on glycemic control especially among COVID-19 patients who also have diabetes. In the COVID-19 era however, getting a good glycemic control is faced with myriads of challenges. These challenges cut across different phases of the infection from a patient living with diabetes who has mild COVID-19 and is being managed at home to a critically ill patient with COVID-19. These challenges need to be identified and elaborated upon so as to device mechanisms to effectively address them.

The aim of this review article is to highlight the various challenges of achieving a good glycemic control among COVID-19 patients who have diabetes. Relevant articles were gotten from PubMed, Google scholar, science direct as well as the grey literature using search terms such as 'diabetes, covid 19', 'glycemic control, covid 19', covid 19, resource-poor settings'.
\end{abstract}

Keywords: COVID-19, challenges, glycemic control, diabetes, resource poor settings

\section{Background}

Coronavirus disease 2019 (COVID-19) is caused by severe acute respiratory syndrome coronavirus 2 (SARS-CoV-2) ${ }^{[1]}$. SARS$\mathrm{CoV}-2$ is a beta coronavirus whose natural reservoir hosts are considered to be bats and wild animals ${ }^{[2]}$. It was first reported by local health authorities who noticed a pattern of pneumonia in a cluster of patients who had been exposed to an animal food market in Wuhan, Hubei Province, China ${ }^{[3]}$. Having met the epidemiological criteria of infecting $>100000$ people in more than 100 countries, COVID-19 was declared as a global pandemic by the World Health Organization (WHO) on March 11, $2020^{[4]}$. The impact of COVID-19 on patients with background co-morbidities is enormous.

The mode of transmission of SARS CoV-2 is primarily through respiratory droplets from one person to another. From the literature, COVID-19 has an incubation period of 2 days to 2 weeks but the median incubation period is 5 days. In terms of clinical presentation, several symptoms have been documented but the most commonly reported symptoms include fever, dry cough, breathlessness, sore throat and some authors have documented abdominal symptoms such as nausea, vomiting, abdominal pain and diarrhoea ${ }^{[3]}$. After the early phase lasting for about 7-10 days, some patients may deteriorate into a more severe form of the disease. This is characterized by sudden deterioration in oxygen saturation and patient may require intensive care unit admission.

Diabetes mellitus is a group of metabolic diseases characterized by chronic hyperglycemia resulting from defects in insulin secretion, insulin action, or both ${ }^{[5]}$. It is characterized by the disorders of carbohydrate, lipid and protein metabolism. Coincidentally, just like COVID-19, diabetes mellitus is also regarded as a pandemic although it is a non-communicable disease [6]. Diabetes is classified into type 1 diabetes, type 2 diabetes, hybrid forms of diabetes, other specific types, hyperglycemia first detected in pregnancy and unclassified diabetes ${ }^{[7]}$. The central theme in diabetes is hyperglycemia. Therefore, glycemic control is an integral part of the management of diabetes mellitus.

People with diabetes develop similar symptoms of COVID19 as patients without diabetes, as stated above. However, some reports of the clinical features of COVID-19 in patients with diabetes have described an initial phase characterized by mild illness. Some authors even documented that in patients with diabetes, fever may not be a prominent symptom in the early phase of COVID-19 in them compared with patients without diabetes who have COVID-19 (59.5\% vs $83.2 \%$; $p=0.02)^{[11]}$. In the later stages of COVID-19 however, in patients with diabetes, the rate of deterioration could be much more faster [11]. Sometimes, deterioration in glycemic control or frank hyperglycaemic emergency is the presenting clinical scenario. 
Some factors have been documented to be associated with a more severe form of COVID-19 and the presence of diabetes mellitus is one of these factors ${ }^{[8,9]}$. A study was done to compare the frequency of diabetes in COVID-19 patients admitted into the intensive care unit (ICU) and those who were not managed inside the ICU. This showed that diabetes mellitus was twice common among the patients admitted into ICU compared with patients who were not ${ }^{[10]}$. Similarly, a larger study done in China recruited 72,314 COVID-19 patients into the study. It was aimed at identifying the factors associated with mortality. Diabetes mellitus was three fold associated with the risk of death $(7.3 \% \text { vs } 2.3 \%)^{[11]}$.

\section{Aim and methods}

The aim of this review article is to highlight the various challenges of achieving a good glycemic control among COVID-19 patients who have diabetes. Relevant articles were gotten from PubMed, Google scholar, Science direct as well as the grey literature using search terms such as 'diabetes, covid 19', 'glycemic control, covid 19', covid 19, resource-poor settings'. The selected articles were adjudged independently to be of adequate quality and relevant to the topic by the authors.

\section{Discussion}

Poor glycemic control is a significant contributor to the risk of having severe COVID-19 or dying from it ${ }^{[12]}$. The hypothesis that has been put forward is the immune suppressive effect of poor glycaemic control. This immunosuppression makes it easy for SARS-CoV-2 to invade the body system and also to suffer from superimposed secondary bacterial infection in the lungs ${ }^{[13]}$. Many diabetes patients have background suboptimal glycaemic control before getting infected by COVID-19 or while some may even have previously undiagnosed diabetes and are just getting diagnosed with diabetes during COVID-19 presentation. Due to different factors, ensuring optimal glycemic control is faced with different challenges among patients with COVID-19 who also have background diabetes.

\section{COVID-19 prevention and the challenge of glycemic control}

As at In several studies on COVID-19, the time of writing this article, the strongest and most effective weapon that society has against this virus is the prevention of its spread ${ }^{[14]}$. The main points in preventing the spread in society are hand hygiene, social distancing, isolation of infected cases and quarantine of contacts or returnees from high risk areas. Epidemiological measures such as lockdown were also put in place.

The infectious nature of COVID-19 and infection prevention measures (as itemized above) reduce the frequency of visits of patients with diabetes to their routine carers such as the General Practitioners, Endocrinologists, Diabetes Educators, Dieticians, Podiatrists and so on ${ }^{[9]}$. This has a huge impact on their glycemic control and general care as the patients with diabetes tend to avoid health care facilities in order to minimize contact with other patients or even health care providers who are assumed to be potentially infected. This is because many clinics in resource poor settings are overcrowded and poorly ventilated. The waiting time before seeing a doctor is usually long due to insufficiency of medical personnel. This is also not unconnected with anxiety and widespread misinformation especially on social media.
Therefore, the impact that the relevant health care providers can make on the glycemic control has been cut off or drastically reduced. Many of these patients have poor glycemic control and would have visited their health care providers but for the fear of contracting COVID-19 ${ }^{[9]}$. There were also the problem of transport facilities due to the lockdown put in place in many countries. In fact, patients with type 1 diabetes have presented with more episodes of diabetic ketoacidosis because they delay presentation at the hospital and tend to wait at home until they deteriorate into frank hyperglycaemic emergency. The delay is partly due to the fear of contracting COVID-19 from healthcare settings ${ }^{[9]}$.

In many countries with adequate health care financing, telemedicine was able to solve some of the challenges ${ }^{[15]}$. It was able to link patients, health care providers and information such as self-monitored blood glucose readings. In resource poor settings however, telemedicine is mostly unavailable or very erratic and this may not be unconnected to availability of internet facilities ${ }^{[15]}$. Also, the majority of the patients in resource poor settings are illiterate and may not be able to use the internet.

\section{Outdoor physical activity and the challenge of glycemic control}

Physical activity involves moving different parts of the body or the whole body with the sole aim of expending energy, whereas exercise is carefully planned, structured and prescribed physical activity ${ }^{[16]}$. Adequate physical activity is essential for good glycemic control, cardiovascular risk reduction and long term wellbeing. However, in order to limit the spread of COVID-19, governments have ordered a series of restrictions and most often than not, physical activity is (was) not allowed outside home in such circumstances ${ }^{[17]}$. These movement restrictions limit outdoor physical activity and exercise which is supposed to help in glycemic control. Some people who are unaware or apparently defy the restrictions often get arrested or get fined which causes emotional distress which is a contributor to worsening glycemic control.

Lack of physical activity can increase insulin resistance which may impair glycemic control. An expected reduction of exercise and an increase of sedentary behavior could, therefore, have a harmful influence on glycemic control ${ }^{[17]}$. A study done in India showed that about $92 \%$ of people living with diabetes reduced their physical activities due to the lockdown restrictions put in place by the national government ${ }^{[18]}$. Decreased physical activity will lower mechanical load. When mechanical load is reduced, metabolic rate decreases and the energy expended declines, This ultimately leads to reduced physical fitness and an energy imbalance (essentially energy surplus) resulting in weight gain ${ }^{[19]}$.

\section{Adherence to sick day rules}

Sick day rules are recommended practices and behaviours that patients with diabetes have been educated to follow in any events of sickness at home. Mild COVID-19 is usually managed at home. However, due to the peculiarities of the patients with diabetes, there are certain rules that need to be followed to ensure adequate glycemic control, prevent ketosis and guarantee general wellbeing, while the acute illness is being managed.

Blood glucose levels tend to rise during an acute illness, such as symptomatic COVID-19, even if the person is not eating and controlling the glucose becomes a challenge. The patient needs 
to check blood glucose more often but this may be a challenge due to the reduced accessibility and increased cost of the glucometer strips, as a result of the lockdown. Adequate hydration and carbohydrate intake are also essential for this kind of patients but these may be difficult for a patient with COVID-19 who has lost appetite as well as taste of delicacy. It has also been reported that adherence to sick day rules in patients with diabetes is difficult and this difficulty is partly related to mental health issues such as depression and anxiety which require dedicated care So, carrying out the sick days plans, which is crucial for out-of-hospital glycemic control during an acute illness, in an event of COVID-19 is also a challenge.

\section{Access to glucose-lowering agents}

Access to glucose lowering medications is also a problem, especially in low income countries that rely heavily on drug importation. It therefore stands to reason that if the drugs could not be accessed, they would not be used and controlling the blood glucose would certainly be a challenge ${ }^{[12]}$. It was reported from a study done in India that Even though, going out to buy medications is an essential activity (in which one could be allowed to go out in the lockdown) many people would still not go out due to the fear of contracting COVID-19 ${ }^{[18]}$.

It has been earlier reported that during global emergencies, insulin production and access reduce significantly and the experience during the COVID-19 global pandemic does not seem to be different ${ }^{[21]}$. This can affect the usage due to poor supply in the markets and economic downturns, especially in nations where paying out of pocket for health care is the norm. Poor usage will translate to suboptimal glycemic control.

\section{Use of glucose-lowering agents}

Use of glucose-lowering agents in a patient with COVID-19 who had background diabetes or is newly diagnosed with diabetes may also be a challenge. Some medications need to be stopped while some will require extra caution to use them. For most patients who buy medications out of pocket, this may be a challenge due to increased cost of care as a result of switch of medications and the need for more glucose checks.

For patients on sodium-glucose transporters-2 (SGLT-2) inhibitors, there is a need to switch from the this group of drugs to another glucose lowering agents during an acute illness to prevent hyperglycemic emergency, specifically, diabetic ketoacidosis ${ }^{[20]}$. Metformin, which is the bedrock of glycemic control in the commonest type of diabetes (type 2 diabetes) may be associated with increased risk of lactic acidosis in the COVID-19 patients with dehydration, sepsis and renal impairment. In moderate to severe COVID-19, there is profound hypoxia of the body tissues which increases the risk of developing lactic acidosis. Glucagonlike peptide receptor-1agonists (GLP-1A agonists) may be suboptimally absorbed from the subcutaneous tissue if the patient is dehydrated due to reduced appetite from the viremic illness.

Generally, the use of Thiazolidinediones (e.g. pioglitazone) is less favored for in-hospital management of acutely ill patients. This is mainly due to the tendency of Thiazolidinediones to cause fluid retention, oedema and suboptimal glycemic control ${ }^{[8]}$. They are also contraindicated in patients with hemodynamic instability, hepatic dysfunction or heart failure and these are conditions that are not uncommonly seen in severe COVID-19 infection. Overall, there are no strict guidelines for the usage of insulin in patients with severe COVID-19 patients. This is also a challenge, especially for the intensivists, in ensuring adequate glycemic control for these patients in whom insulin resistance is prominent.

\section{Use of other drugs that can affect glucose control}

Hydroxychloroquine is still being studied in some trials as a potential therapeutic agent for COVID-19, after some initial encouraging results ${ }^{[22]}$. It is however a challenge for patients on sulphonylureas because of the increased risk of hypoglycemia, especially in a patient with reduced food intake or vomiting due to the illness ${ }^{[23]}$.

For most patients with diabetes, like the general population, symptoms of COVID-19 are usually mild. Patients may take acetaminophen (paracetamol) just for control of symptoms. However, the sensitivity of some chronic glucose monitors (CGM) monitors is affected by paracetamol ${ }^{[8]}$. In such situations, use of glucometers may be considered. The challenge is in the fact that patient may be unaware of this interference and this may prevent or delay necessary intervention.

Some studies have suggested the beneficial roles of intravenous steroids in alleviating morbidity and mortality in critically ill COVID-19 patients ${ }^{[24]}$. However, in the presence of diabetes, this increases insulin resistance and poses a risk of worsening glycemic control.

Some treatment protocols have advocated for the use of high dose intravenous vitamin $\mathrm{C}$ in the management of COVID-19 patients admitted into the intensive care unit (ICU) ${ }^{[27]}$. Such a high dose of ascorbic acid can give a falsely elevated capillary glucose when measured with a glucometer ${ }^{[28]}$. This may mislead the physicians resulting to increased dosage of insulin and attendant hypoglycemia, of varying severity.

\section{Access to food and health eating}

Many nations are implementing measures to reduce the spread of COVID-19. These measures include self-quarantine and lockdowns where businesses are temporarily closed down. Studies have suggested that these measures may affect normal food-related practices ${ }^{[25]}$. In some countries, there are restrictions on the activities of restaurants and places that offer take-away packs. Lockdowns have also affected the transport of essential food items from rural/farmlands to urban areas. So, farm products such as fruits and vegetables tend to perish and those who eventually make it to the urban areas are usually expensive.

It is well known that dietary modification is key to controlling glucose excursions in patients with diabetes. Maintaining a healthy diet can be a challenge under the aforementioned circumstances. In the absence of healthy diet, glycemic patterns may be suboptimal even with medication adherence. Being at home for extended periods, especially without company or with limited activities can also lead to overeating which can also make glycemic control challenging ${ }^{[25]}$. It is therefore advised that patients with diabetes seek guidance on what constitutes healthy portions from licensed dieticians preferably via the internet. However, in a place where telemedicine does not work, getting a consultation with the dietician is a challenge too considering the fear of not wanting to go the health facility so as not to contract COVID-19. Moreover, feeding in some isolation centers do not take the dietary peculiarities of patients with diabetes into consideration

There is no gainsaying that good and optimal nutrition is essential for health. This is even more relevant in patients with 
diabetes. Moreover, the immune system requires adequate supply of nutrients to fight pathogens such as SARS CoV-2. Lockdown and other government measures put in place to curtail the spread of the virus limit food accessibility. Consequently, limited access health diet may lead to an increased consumption of highly processed foods, which tend to be high in fats, sugars and salt. The overall effect of this is poor glycemic control and suboptimal general wellbeing.

\section{Challenges with glucose monitoring}

Regular monitoring of blood glucose is considered central in the management of diabetes. Glucose meters being used at home may also be used by patients with diabetes who are hospitalized due to COVID-19 to check their own blood glucose levels and provide the readings to the health care personnel caring for them. However, if the patient is severely ill, this may constitute a challenge and make monitoring of blood glucose inadequate. Also in many centers, provision of personal protective equipment is not adequate resulting in less frequent reviews and inadequate glucose checks by the medical staff.

Once glucose monitoring is inadequate, optimal glycemic control remains a challenge. In some isolation wards, anything, including a glucometer that enters the room, will be discarded at discharge. Patients may not be able to afford another glucometer in the immediate post-discharge period and glucose monitoring may be challenging.

Due to the lockdowns put in place by the governments, patients using glucometers may have difficulty accessing the accessories such as strips or batteries and when there is a need for a repair or replacement, this may be difficult. This will go a long way in affecting glucose monitoring thereby impairing the glucose control.

Concerning CGM, it has been reported that hypoxia and reduced tissue perfusion in COVID-19 patients with severe disease may lead to inaccurate results using the apparatus. ${ }^{[15]}$

\section{Conclusion}

This review has highlighted the challenges facing an integral part of management of people living with diabetes who get infected with COVID-19 as they are at increased risk of morbidity and mortality. Hyperglycemia is a principal factor that must be controlled in diabetes. Controlling glucose however has peculiar challenges which are in various forms. Recognizing these challenges are of critical importance so as to be able to combat them.

\section{Ethics approval and consent to participate}

Not applicable.

\section{List of abbreviations}

CGM - Continuous glucose monitors

COVID - 19 - Coronavirus disease- 2019

GLP-1 - Glucagon-like peptide receptor-1

ICU - Intensive care unit

SARS - CoV-2 - Severe acute respiratory syndrome coronavirus 2

SGLT - 2 - Sodium-glucose transporters-2

WHO - World Health Organization

\section{Data availability}

Not applicable

\section{Conflicts of Interest}

None

\section{Funding Statement}

Self-funded

\section{Authors' contributions}

EA was involved in the conception of the research idea and editing of the manuscript. ATA was involved in the conception of the idea and writing of the manuscript.

\section{References}

[1] Anjorin AA. The coronavirus disease 2019 (COVID-19) pandemic: A review and an update on cases in Africa. Asian Pac J Trop Med. 2020; 13:199-203.

[2] Cui J, Li F, Shi Z. Origin and evolution of pathogenic coronaviruses. Nat Rev Microbiol. 2019; 17:181-192.

[3] Zhu N, Zhang D, Wang W. A novel coronavirus from patients with pneumonia in China, $2019 \mathrm{~N}$ Engl J Med. 2020; 382:727-733

[4] Ohia C, Bakarey AS, Ahmad T. COVID-19 and Nigeria:putting the realities in context. Int J Infect Dis, 2020; 95: 279-285.

[5] Kharroubi AT, Darwish HM. Diabetes mellitus: The epidemic of the century. World J. Diabetes. 2015; 6(6): 850-867.

[6] Narayan KMV. Diabetes Pandemic: looking for the silver lining. Clin Diabetes. 2005; 23(2): 51-52.

[7] World Health Organization. Classification of diabetes mellitus 2019. WHO Geneva. 2020: p12

[8] Singh AK, Gupta R, Ghosh A, Misra A. Diabetes in COVID-19: Prevalence, pathophysiology, prognosis and practical considerations. Diabetes Metab Syndr. 2020;14: 303-310.

[9] Scott ES, Jenkins AJ, Fulcher GR. Challenges of diabetes management during the COVID-19 pandemic. Med. J. Australia. 2020; doi:10.5694/mja2.50665.

[10] Guan WJ, Ni ZY, Hu Y, et al. Clinical characteristics of coronavirus disease 2019 in China. N Engl J Med. 2020; doi: 10.1056/NEJMoa2002032.

[11] Wu Z, McGoogan JM. Characteristics of and important lessons from the coronavirus disease 2019 (COVID-19) outbreak in China: summary of a report of 72314 cases from the Chinese Center for Disease Control and Prevention. JAMA. 2020; 323(13): 1239.

[12] Gamble A, Pham Q, Goyal S, Cafazzo J. The challenges of COVID-19 for people living with diabetes: considerations for digital health. JMIR Diabetes. 2020;5 (2): e19581.

[13] Singh AK, Gupta R, Ghosh A, Misra A. Diabetes in COVID-19: Prevalence, pathophysiology, prognosis and practical considerations. Diabetes Metab Syndr. 2020;14:303-310.

[14] Guner R, Hasanoglu I, Aktas F. COVID-19: Prevention and control measures in community. Turk J Med Ski. 2020; 50(3): 571-577. 
[15] Song X, Liu X, Wang C. The role of telemedicine during the COVID-19 epidemic in China-experience from Shandong province. Critical care. 2020; 24: 178.

[16] Colberg SR, Signal RJ, Yardley JE, Riddell MC, Dunstan DW, Dempsey PC. Physical Activity/Exercise and Diabetes: A Position Statement of the American Diabetes Association. Diabetes Care 2016; 39 (11): 2064-2079.

[17] Tornese G, Ceconi V, Monasta L, Carletti C, Faleschini E, Barbi E. Glycemic Control in Type 1 Diabetes Mellitus During COVID-19 Quarantine and the Role of In-Home Physical Activity. Diabetes Technol. Ther. 2020; 22(6): doi.org/10/10.1089/dia.2020.0169.

[18] Pal R, Yadav U, Verma A, Bhadada SK. Awareness regarding COVID-19 and problems being faced by young adults with type 1 diabetes mellitus amid nationwide lockdown in India: A qualitative interview study. Primary Care Diabetes. 2020; doi.org/10/1016/j.pcd.2020.07.001

[19] Jakobsson J, Malm C, Furberg M, Ekelund U, Svensson M. Physical Activity During the Coronavirus (COVID19) Pandemic: Prevention of a Decline in Metabolic and Immunological Functions. Frontiers. 2020; doi.org/10.3389/fspor.2020.00057.

[20] Pal R, Bhadada SK. Should anti-diabetic medications be reconsidered amid COVID-19 pandemic? Diabetes Res Clin Pract. 2020;163:108146.

[21] Cefalu WT, Dawes DE, Gavlak G, Goldman D, Herman WH, Van Nuys K, Powers AC, Taylor SI, Yatvin AL,
Insulin Access and Affordability Working Group Insulin access and affordability working group: conclusions and recommendations. Diabetes Care. 2018;41(6):12991311.

[22] Arshad S, Kilgore P, Chaudhry ZS, O,Neill W, Zervos M. Treatment with Hydroxychloroquine, Aithromycin and Combination in Patients with COVID-19. Int J Infect Dis. 2020; doi.org/10.1016/j/ijid.2020.06.099.

[23] Gupta R, Ghosh A, Singh AK, Misra A. Clinical considerations for patients with diabetes in times of COVID-19 epidemic. Diabetes Metab Syndr. 2020; 14(3):211-212.

[24] Veronese N, Demurtas J, Yang L, Tonelli R, Pizzol D, Zou L, Tully MA, et al. Use of Corticosteroids in Coronavirus Disease 2019 Pneumonia: A Systematic Review of the Literature. Front. Med., 24 April 2020 | https://doi.org/10.3389/fmed.2020.00170

[25] World Health Organization. Food and nutrition tips during quarantine. WHO, Geneva. 2020.

[26] Hamdy O, Gabbay RA. Early observation and mitigation of challenges in the diabetes management of COVID-19 patients in critical care units. Diabetes Care. 2022; doi.org/10.2337/dc20-0944.

[27] Hemila H, Chalker E. Vitamin C can shorten the length of stay in the ICU-Meta-analysis. Nutrients. 2019; 11:708.

[28] Cho J, Ahn S, Yim J et al. Influence of vitamin C and maltose on the accuracy of three models of glucometers. Ann Lab Med. 2016; 36:271-274. 